\title{
Enlarged subarachnoid spaces and intracranial hemorrhage in children with accidental head trauma
}

\author{
Amanda K. Fingarson, DO, ${ }^{1}$ Maura E. Ryan, MD, ${ }^{2}$ Suzanne G. McLone, MPH, ${ }^{3}$ \\ Corey Bregman, MD, ${ }^{2}$ and Emalee G. Flaherty, MD'
}

Departments of ${ }^{1}$ Pediatrics, Division of Child Abuse Pediatrics, and 2 Medical Imaging, Division of Neuroradiology; and ${ }^{3}$ Stanley Manne Children's Research Institute, Ann and Robert H. Lurie Children's Hospital of Chicago, Illinois

\begin{abstract}
OBJECTIVE Benign external hydrocephalus (BEH) is an enlargement of the subarachnoid spaces (SASs) that can be seen in young children. It is controversial whether children with $\mathrm{BEH}$ are predisposed to developing subdural hemorrhage (SDH) with or without trauma. This issue is clinically relevant as a finding of unexplained SDH raises concerns about child abuse and often prompts child protection and law enforcement investigations.

METHODS This retrospective study included children (1-24 months of age) who underwent head CT scanning after an accidental fall of less than 6 feet. Head CT scans were reviewed, cranial findings were documented, and the SAS was measured and qualitatively evaluated. Enlarged SAS was defined as an extraaxial space (EAS) greater than $4 \mathrm{~mm}$ on CT scans. Clinical measurements of head circumference $(\mathrm{HC})$ were noted, and the head circumference percentile was calculated. The relationship between enlarged SAS and HC percentile, and enlarged SAS and intracranial hemorrhage $(\mathrm{ICH})$, were investigated using bivariate analysis.
\end{abstract}

RESULTS Of the 110 children included in this sample, 23 had EASs greater than $4 \mathrm{~mm}$. The mean patient age was 6.8 months (median 6.0 months). Thirty-four patients (30.9\%) had ICHs, including subarachnoid/subpial (6.2\%), subdural $(6.2 \%)$, epidural (5.0\%), and unspecified extraaxial hemorrhage (16.5\%). Enlarged SAS was positively associated with subarachnoid/subpial hemorrhage; there was no association between enlarged SASs and either SDH or epidural hemorrhage. A larger SAS was positively associated with larger $\mathrm{HC}$ percentile; however, $\mathrm{HC}$ percentile was not independently associated with $\mathrm{ICH}$.

CONCLUSIONS Enlarged SAS was not associated with SDH, but was associated with other ICHs. The authors' findings do not support the theory that BEH predisposes children to SDH with minor accidental trauma.

https://thejns.org/doi/abs/10.3171/2016.8.PEDS16146

KEY WORDS intracranial hemorrhage; enlarged subarachnoid spaces; benign external hydrocephalus; trauma

$\mathrm{B}$ ENIGN external hydrocephalus (BEH) is an enlargement of the subarachnoid space (SAS) that is seen in some young children. It is frequently associated with macrocephaly and is typically characterized by a period of rapidly increasing head circumference (HC) in infancy that resolves by 2 years of age. ${ }^{8,15,28}$ This condition is known by multiple terms, including benign extraaxial fluid, benign subdural collections of infancy, benign enlargement of the SASs, and benign extracerebral fluid collections in infancy. There are no established criteria for diagnosis, although several studies have used extraaxial space (EAS) measurements greater than $4 \mathrm{~mm}$ on head CT scans to identify BEH. 2,6,11,13,19 It is unclear whether young children with enlarged SASs are at risk for the development of subdural hemorrhage (SDH) with minor trauma or no trauma. Some case series and a mathematical model by Papasian and Frim have concluded that children with enlarged SASs are at increased risk of SDH due to stretched bridging veins. . $^{3,10,13,16,18,21}$ However, a finite element model by Raul et al. concluded the opposite: that increased cere-

ABBREVIATIONS $\mathrm{BEH}=$ benign external hydrocephalus; $\mathrm{CPS}=$ child protective services; $\mathrm{EAH}(\mathrm{NOS})=$ extraaxial hemorrhage (not otherwise specified); EAS = extraaxial space; $E D H=$ epidural hemorrhage; $\mathrm{HC}=$ head circumference; $\mathrm{ICH}=$ intracranial hemorrhage; $\mathrm{SAH}=$ subarachnoid hemorrhage; $\mathrm{SAS}=$ subarachnoid space; $\mathrm{SDH}=$ subdural hemorrhage; $\mathrm{SPH}=$ subpial hemorrhage.

SUBMITTED April 7, 2016. ACCEPTED August 31, 2016.

INCLUDE WHEN CITING Published online November 25, 2016; DOI: 10.3171/2016.8.PEDS16146. 
bral spinal fluid in the SASs creates a damping effect and protects against intracranial hemorrhage (ICH) ${ }^{20}$ Studies that have looked at larger groups of children with enlarged SASs have concluded that subdural collections are infrequently found. .,12,24 $^{2}$

This issue becomes important when evaluating children for possible maltreatment. Subdural hemorrhages are sometimes found on head CT or brain MR images of infants who present for evaluation for macrocephaly or for another cause. Given that physical abuse is considered to be the most common cause of SDH in this age group, this finding presents clinicians with a diagnostic challenge. ${ }^{1,17,23,25}$ Clinicians must determine whether the collections represent hemorrhage from a remote inflicted injury (where the other signs of injury, such as fractures, bruises, or retinal hemorrhages, may have resolved) or an underlying condition that predisposed the patient to SDH in the absence of either inflicted or significant trauma.

This study aims to determine whether children with enlarged SASs, measured quantitatively by CT scans as EAS greater than $4 \mathrm{~mm}$ and assessed qualitatively, are more likely to develop SDH or other ICH after minor accidental head trauma, i.e., falls from less than 6 feet. In addition, we assessed agreement between quantitative measurements of SAS size and the neuroradiologists' qualitative assessment of SAS enlargement. The systematic measurements of SAS sizes in infants and young children collected during this study will assist us in evaluating imaging standards for diagnosing BEH.

\section{Methods \\ Study Participants}

Study participants included children 1-24 months of age who presented to a large Midwestern pediatric hospital with reported accidental falls from less than 6 feet and underwent head CT scanning between June 1, 2006, and June 30, 2012. Study participants were identified using the hospital's child abuse team's database. Additional cases were identified through a search of the hospital's electronic medical records (Epic) using the following ICD-9-CM diagnosis codes: 782.2, 784.2, 800-804, 850-854, 873, 900, 910, 920, 925, 959.01, and 959.09. The following E codes were also searched for via Epic: E880-E886 and E888. Institutional review board approval was obtained for this study.

Children were excluded if the neuroradiologists (M.E.R. and C.B.) determined that the image quality was insufficient due to motion, artifact, slice selection, or angulation that precluded accurate measurement of EAS. Infants younger than 1 month of age were excluded because birth trauma could have contributed to any intracranial findings. Children were also excluded if no approximate time or date of trauma was known or documented, or if more than one trauma history was provided. Children with radiographic concern for prior injury or an underlying neurological diagnosis were excluded. Finally, if the hospital's child abuse team suspected the child was a victim of abuse or neglect, the case was excluded because the caregivers may have provided inaccurate information about the time and nature of the trauma.
Children were included in the study if they were transferred from an outside hospital that referred to state child protective services (CPS), but our hospital's child abuse team concluded they did not suspect the injury was caused by abuse or neglect. These children were included because some hospitals have protocols stating that they must report all infant head trauma to CPS regardless of their suspicion for abuse. Per our institution's protocol, the hospital's multidisciplinary child abuse team of child abuse pediatricians and social workers is consulted on all head injuries in children younger than 2 years of age, head injuries at any age that have been reported to CPS, and any time clinicians have concern for maltreatment. The team's social workers conduct detailed psychosocial assessments on these cases and document detailed information about the reported trauma history. This multidisciplinary team guides decisions about reporting to state CPS.

\section{Data Abstraction}

Clinical information was reviewed by a board-certified child abuse pediatrician (A.K.F.). Information obtained included the child's age, gestational age at birth, trauma history details, clinical symptoms, physical examination findings, and duration of time from trauma to examination/imaging. If clinical $\mathrm{HC}$ measurements and the patient's weight were obtained within a 2-week period of the head CT, those measurements were recorded. The fall height was estimated using the following guidelines: falls from the child's height, couches, standard chairs, and standard beds were estimated to be less than 3 feet; falls from caregivers' arms, high chairs, cribs, tall beds, tables, and countertops were estimated to be 3-6 feet.

\section{Evaluation of Images}

Head CT images were reviewed by one of 2 boardcertified neuroradiologists (M.E.R. or C.B.). Questionable findings were decided by consensus. Information regarding skull fractures and parenchymal injury was recorded. Intracranial hemorrhage (ICH) was categorized as epidural hemorrhage (EDH), subdural hemorrhage (SDH), subarachnoid/subpial hemorrhage (SAH/SPH), or extraaxial hemorrhage (not otherwise specified) (EAH [NOS]). The unspecified EAH category was used when hemorrhages were too small to accurately distinguish between SDH and $\mathrm{EDH}$. The 2 neuroradiologists, who were blinded to each other's judgment, also qualitatively classified the subarachnoid spaces by the degree of enlargement (none, borderline, mild, moderate, or severe).

Head size was calculated from CT using equations described in previous publications. HC was estimated by measuring the anteroposterior and transverse dimensions on axial head CT scans at the level of the basal ganglia, and area was approximated by using an ellipse equation: Area $=\pi \times($ transverse $/ 2) \times($ anteroposterior $/ 2) .{ }^{5}$

Subarachnoid space (estimated by EAS size) is defined as the greatest distance from the gyral surface to the inner calvarial cortex. EAS was measured on axial CT scans at the level of the lateral ventricle and averaged between the left and right sides (Fig. 1). ${ }^{9}$

Head CT scans obtained at outside hospitals in chil- 


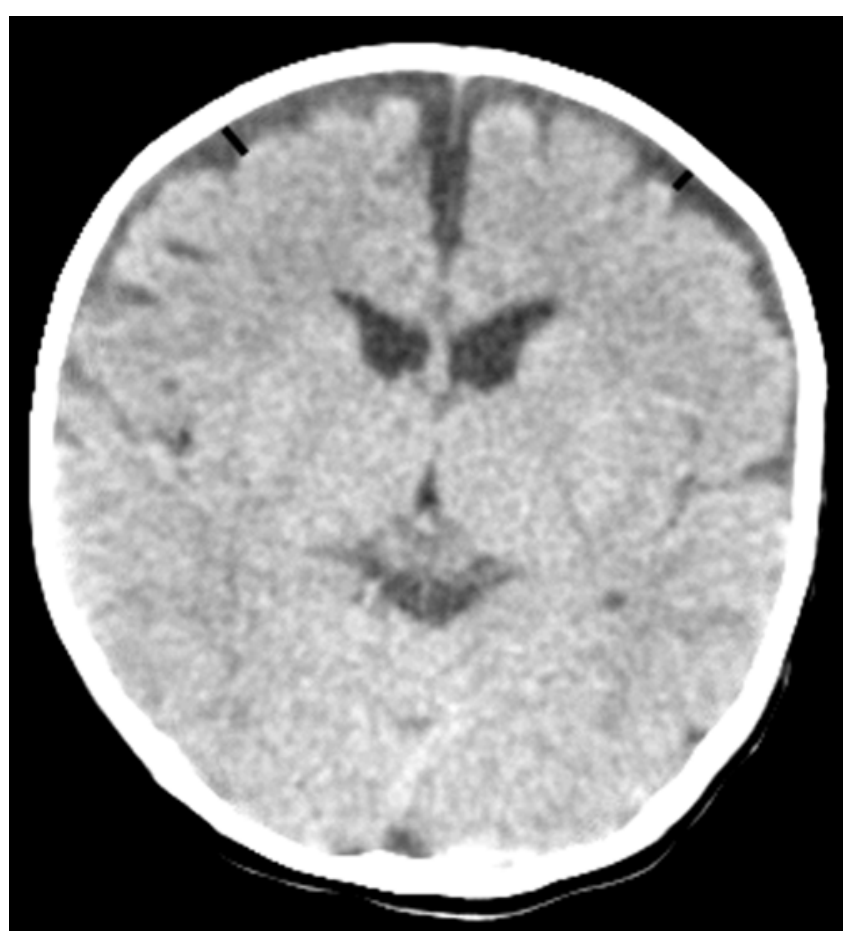

FIG. 1. Estimation of SAS from the measurement of EAS on axial CT scans. EAS size is measured as the greatest distance from the frontal gyral surface to the inner calvarial cortex (black lines).

dren who were transferred to our institution were included if the studies were loaded into our hospital's picture archiving and communication system, and if the neuroradiologist determined that the imaging was of sufficient quality for interpretation.

\section{Statistical Analysis}

Analyses were performed using the SPSS software package (version 22.0, IBM); p values $<0.05$ were considered statistically significant. Standard statistical methods were used to summarize the data: frequencies and percentages for nominally scaled variables, and means and standard deviations for continuously scaled variables. Statistical differences between groups were calculated using the Student t-test for continuous measurements and Fisher's exact test for categorical variables. Statistical correlations between continuous measurements were calculated using Pearson's r. An interrater reliability analysis using the kappa statistic was performed to determine consistency among raters.

\section{Results}

Of the 136 patients identified who had undergone head CT scanning for a fall, 4 were excluded due to excessive fall distance ( $>6$ feet), 4 were excluded for unexplained bruises possibly caused by abuse, 4 were excluded for unclear timing or mechanism of injury, and 14 were excluded due to neuroradiology limitations.

Our final cohort included 110 children, with a mean age of 6.8 months (median age 6.0 months; Table 1). The population was predominantly white and Hispanic $(42.7 \%$
TABLE 1. Study population characteristics for 110 children with accidental head trauma

\begin{tabular}{|c|c|}
\hline Characteristic & Value* $^{*}$ \\
\hline Mean age in mos ( \pm SD) & $6.8 \pm 4.65$ \\
\hline \multicolumn{2}{|l|}{ Sex } \\
\hline Male & $56(50.9)$ \\
\hline Female & $54(49.1)$ \\
\hline \multicolumn{2}{|l|}{ Race/ethnicity } \\
\hline White/Caucasian & $47(42.7)$ \\
\hline Hispanic/Latino & $41(37.3)$ \\
\hline Asian & $5(4.5)$ \\
\hline Black & $3(2.7)$ \\
\hline Other/unknown/declined & $14(12.7)$ \\
\hline \multicolumn{2}{|l|}{ Insurance $(n=109)$} \\
\hline Private & $63(57.8)$ \\
\hline Public & $46(42.2)$ \\
\hline \multicolumn{2}{|l|}{ Gestational age at birth $(n=87)$} \\
\hline Term & $74(85.1)$ \\
\hline Preterm & $13(14.9)$ \\
\hline Mean EAS measurement in $\mathrm{mm}( \pm \mathrm{SD})$ & $2.9 \pm 1.71$ \\
\hline$>4 \mathrm{~mm}$ & $23(20.9)$ \\
\hline \multicolumn{2}{|l|}{ EAS qualitative assessment } \\
\hline No enlargement & $70(63.6)$ \\
\hline Borderline enlargement & $20(18.2)$ \\
\hline Mild enlargement & $13(11.8)$ \\
\hline Moderate enlargement & $7(6.4)$ \\
\hline Severe enlargement & $0(0)$ \\
\hline \multicolumn{2}{|l|}{ Intracranial injuries } \\
\hline Fracture & $85(77.3)$ \\
\hline $\mathrm{ICH}$ & $34(30.9)$ \\
\hline $\mathrm{SAH} / \mathrm{SPH}(\mathrm{n}=81) \dagger$ & $5(6.2)$ \\
\hline $\mathrm{SDH}(n=81) \ddagger$ & $5(6.2)$ \\
\hline EAH (NOS) $(n=91) \S$ & $15(16.5)$ \\
\hline $\mathrm{EDH}(\mathrm{n}=80) \uparrow$ & $4(5.0)$ \\
\hline Multiple ICHs $(n=81)^{\star *}$ & $5(6.2)$ \\
\hline
\end{tabular}

* Values are presented as the number of patients (\%) unless otherwise indicated. Percentages may not add up to $100 \%$ due to rounding.

$\dagger, \ddagger, \S, \uparrow,{ }^{* *}$ Categories are mutually exclusive.

and $37.3 \%$, respectively). Males and females were equally represented $(50.9 \%$ and $49.1 \%$, respectively). Most of the children in the study were born at term. Of the 82 children with gestational age documented in the medical record, only $14.9 \%$ were born before 37 weeks' gestation. The average age of the premature subjects $(n=13)$ was 34.3 weeks. Only 1 child was born prior to 32 weeks' gestation. More than half of the patients were privately insured. Of the 110 eligible children, 34 (30.9\%) had ICH. ICH types included about equal numbers of SAH/SPH, SDH, and EDH. Almost half (15) had EAH (NOS) (16.5\%), and 5 children $(6.2 \%)$ had more than 1 type of ICH. Skull fractures were very common in our sample $(85 / 110,77.3 \%$; Table 1).

The overall mean EAS measurement was $2.9 \mathrm{~mm}$ (me- 
dian $2.6 \mathrm{~mm}$; Table 1). Twenty-three participants in the sample (20.9\%) had an EAS size greater than $4 \mathrm{~mm}$. The qualitative assessment determined that most of the sample (70/110) did not have any enlargement of the EAS (63.6\%), while $20(18.2 \%)$ had borderline enlargement, $13(11.8 \%)$ had mild enlargement, and 7 (6.4\%) had moderate enlargement. No children had severe enlargement (Table 1). There was no association between age and EAS size. There was a positive, significant association between preterm birth and enlarged EAS in the sample-31\% of preterm births had enlarged EAS versus $15 \%$ who were not preterm births.

EAS qualitative estimates made by 2 neuroradiologists $\left(\mathrm{EAS}_{1}\right.$ and $\left.\mathrm{EAS}_{2}\right)$ were positively correlated with the EAS quantitative measurements. The interrater reliability for the 2 neuroradiologists was found to be $\kappa=0.46$ ( $p<$ 0.001 , which indicates moderate agreement.

Thirty-seven children in the sample (33.6\%) had an HC measurement documented. The mean $\mathrm{HC}$ percentile was 53rd percentile and the median was $63 \mathrm{rd}$ percentile. This was similar to the weight percentiles (mean 54th and median 57th percentiles) in our population. There was poor agreement between clinically measured and radiographically calculated head circumferences, so only clinically measured HCs were used.

The mean EAS was significantly larger in those with $\mathrm{SAH} / \mathrm{SPH}(5.4 \mathrm{~mm}$ vs $2.6 \mathrm{~mm}, \mathrm{p}<0.001)$ and those with multiple hemorrhages $(4.1 \mathrm{~mm}$ vs $2.6 \mathrm{~mm}, \mathrm{p}<0.05)$. The mean EAS was also significantly higher in those with ICH overall (all ICH types combined [3.6 mm vs $2.6 \mathrm{~mm}$, $\mathrm{p}<0.05]$ ). There was no association between increased EAS and SDH, EAH (NOS), or EDH (Table 2). There was no difference in the rate of ICH in children who fell less than 3 feet versus 3-6 feet. Although the number of patients became very small, fall height also did not change the outcomes. Both SAH/SPH and multiple hemorrhages remained associated with enlarged EAS. Increased EAS was positively associated with larger clinically measured HC percentile $(r=0.44, p<0.01)$. However, clinically measured $\mathrm{HC}$ alone was not associated with increased EAS $(r=0.16, p \geq 0.05)$.

\section{Discussion}

Although some studies have suggested that an enlarged SAS predisposes an infant to develop SDH after minor head trauma, this study found no such association. We did find that increased SAS size was associated with ICH (overall), more specifically SAH/SPH and multiple ICHs. Macrocrania alone was not associated with an increased incidence of $\mathrm{ICH}$, a finding that is supported by the literature. ${ }^{26}$

Overall, our population had a higher incidence of ICH and skull fractures than described in other studies of falls from a lower height. ${ }^{14,22,27}$ This is not unexpected since we identified subjects primarily through the child abuse team consult database, with supplemental cases identified through billing codes focused on head injuries with falls. While the hospital protocol requires all children younger than 2 years with head injury to undergo a child abuse team consult, our methods would not be expected to capture all children who presented to our institution after a
TABLE 2. EAS size and ICH

\begin{tabular}{|c|c|c|}
\hline ICH Type & Mean EAS, mm (SD) & $\%$ EAS $>4 \mathrm{~mm}$ \\
\hline \multicolumn{3}{|l|}{ SAH/SPH $(n=81)$} \\
\hline Yes & $5.4(0.8)^{*}$ & $100^{*}$ \\
\hline No & $2.6(1.5)$ & 13.2 \\
\hline \multicolumn{3}{|l|}{$\mathrm{SDH}(n=81)$} \\
\hline Yes & $2.7(1.1)$ & 0.0 \\
\hline No & $2.6(1.5)$ & 13.2 \\
\hline \multicolumn{3}{|c|}{$\mathrm{EAH}(\mathrm{NOS})(\mathrm{n}=91)$} \\
\hline Yes & $3.3(2.2)$ & 20.0 \\
\hline No & $2.6(1.5)$ & 13.2 \\
\hline \multicolumn{3}{|l|}{$\mathrm{EDH}(n=80)$} \\
\hline Yes & $2.8(2.1)$ & 25.0 \\
\hline No & $2.6(1.5)$ & 13.2 \\
\hline \multicolumn{3}{|c|}{ Multiple ICHs $(n=81)$} \\
\hline Yes & $4.1(1.2) \dagger$ & $80.0 \dagger$ \\
\hline No & $2.6(1.5)$ & 13.2 \\
\hline \multicolumn{3}{|c|}{ All ICH Types $(n=110)$} \\
\hline Yes & $3.6(1.9) \dagger$ & $38.2 \dagger$ \\
\hline No & $2.6(1.5)$ & 13.2 \\
\hline
\end{tabular}

short fall and underwent neuroimaging but did not have an identified head injury.

While a portion of our sample had large HCs, the majority of our sample consisted of children with average HCs. Similarly, most did not have BEH, when using the definition of EAS greater than $4 \mathrm{~mm}$. However, the number of children who did have EAS greater than $4 \mathrm{~mm}$ (21\%) was somewhat higher than expected. This suggests that either the condition is quite common or a higher cutoff in defining BEH may be justified.

Another limitation of the study is its retrospective design, which relied on information documented in the medical record. In addition, caregivers may have provided vague or false details about the trauma. It is possible that some children with inflicted injuries may have been unintentionally included in this study, and some children with accidental injuries may have been wrongly excluded. This concern is somewhat mitigated by our institution's protocol mandating thorough social work assessments and child abuse pediatrician consults on children younger than 2 years with head injuries. In addition, variations in the degree of angulation of the CT scans potentially impacted measurements of SAS, although this limitation was minimized by only including children who had CT scans that were determined by a neuroradiologist to be of sufficient quality for interpretation. The size of our study population is another limitation. While there were 110 children in the study, the numbers in the ICH subgroups were small.

While this study represents an important first step in evaluating the relationship between SAS size and SDH in the setting of accidental falls from a low height, larger prospective studies are needed. There is also a need to study children with markedly enlarged SAS to determine whether our results are replicated in this population. 


\section{Conclusions}

Large SAS size was not associated with SDH, but it was associated with SAH/SPH after trauma. While children with larger SASs tended to have larger heads, increased $\mathrm{HC}$ was not independently associated with an increase in $\mathrm{SAH} / \mathrm{SPH}$. Our findings do not support the hypothesis that $\mathrm{BEH}$ predisposes children to SDH following minor accidental trauma, although the condition may predispose to $\mathrm{SAH} / \mathrm{SPH}$.

\section{Acknowledgments}

We thank Stacey C. Tobin, $\mathrm{PhD}$, for manuscript review and editorial support.

\section{References}

1. Duhaime AC, Alario AJ, Lewander WJ, Schut L, Sutton LN, Seidl TS, et al: Head injury in very young children: mechanisms, injury types, and ophthalmologic findings in 100 hospitalized patients younger than 2 years of age. Pediatrics 90:179-185, 1992

2. Fukuyama Y, Miyao M, Ishizu T, Maruyama H: Developmental changes in normal cranial measurements by computed tomography. Dev Med Child Neurol 21:425-432, 1979

3. Ghosh PS, Ghosh D: Subdural hematoma in infants without accidental or nonaccidental injury: benign external hydrocephalus, a risk factor. Clin Pediatr (Phila) 50:897-903, 2011

4. Greiner MV, Richards TJ, Care MM, Leach JL: Prevalence of subdural collections in children with macrocrania. AJNR Am J Neuroradiol 34:2373-2378, 2013

5. Hahn FJ, Chu WK, Cheung JY: CT measurements of cranial growth: normal subjects. AJR Am J Roentgenol 142:12531255,1984

6. Hellbusch LC: Benign extracerebral fluid collections in infancy: clinical presentation and long-term follow-up. J Neurosurg 107 (2 Suppl):119-125, 2007

7. Kapila A, Trice J, Spies WG, Siegel BA, Gado MH: Enlarged cerebrospinal fluid spaces in infants with subdural hematomas. Radiology 142:669-672, 1982

8. Kleinman PK, Zito JL, Davidson RI, Raptopoulos V: The subarachnoid spaces in children: normal variations in size. Radiology 147:455-457, 1983

9. Lam WW, Ai VH, Wong V, Leong LL: Ultrasonographic measurement of subarachnoid space in normal infants and children. Pediatr Neurol 25:380-384, 2001

10. Laubscher B, Deonna T, Uske A, van Melle G: Primitive megalencephaly in children: natural history, medium term prognosis with special reference to external hydrocephalus. Eur J Pediatr 149:502-507, 1990

11. Libicher M, Tröger J: US measurement of the subarachnoid space in infants: normal values. Radiology 184:749-751, 1992

12. McKeag H, Christian CW, Rubin D, Daymont C, Pollock AN, Wood J: Subdural hemorrhage in pediatric patients with enlargement of the subarachnoid spaces. J Neurosurg Pediatr 11:438-444, 2013

13. McNeely PD, Atkinson JD, Saigal G, O'Gorman AM, Farmer JP: Subdural hematomas in infants with benign enlargement of the subarachnoid spaces are not pathognomonic for child abuse. AJNR Am J Neuroradiol 27:1725-1728, 2006

14. Murray JA, Chen D, Velmahos GC, Alo K, Belzberg H, Asensio JA, et al: Pediatric falls: is height a predictor of injury and outcome? Am Surg 66:863-865, 2000

15. Nickel RE, Gallenstein JS: Developmental prognosis for infants with benign enlargement of the subarachnoid spaces. Dev Med Child Neurol 29:181-186, 1987
16. Papasian NC, Frim DM: A theoretical model of benign external hydrocephalus that predicts a predisposition towards extra-axial hemorrhage after minor head trauma. Pediatr Neurosurg 33:188-193, 2000

17. Parent AD: Pediatric chronic subdural hematoma: a retrospective comparative analysis. Pediatr Neurosurg 18:266271, 1992

18. Piatt JH Jr: A pitfall in the diagnosis of child abuse: external hydrocephalus, subdural hematoma, and retinal hemorrhages. Neurosurg Focus 7(4):e4, 1999

19. Prassopoulos P, Cavouras D, Golfinopoulos S, Nezi M: The size of the intra- and extraventricular cerebrospinal fluid compartments in children with idiopathic benign widening of the frontal subarachnoid space. Neuroradiology 37:418421, 1995

20. Raul JS, Roth S, Ludes B, Willinger R: Influence of the benign enlargement of the subarachnoid space on the bridging veins strain during a shaking event: a finite element study. Int J Legal Med 122:337-340, 2008

21. Ravid S, Maytal J: External hydrocephalus: a probable cause for subdural hematoma in infancy. Pediatr Neurol 28:139141,2003

22. Trenchs V, Curcoy AI, Morales M, Serra A, Navarro R, Pou J: Retinal haemorrhages in head trauma resulting from falls: differential diagnosis with non-accidental trauma in patients younger than 2 years of age. Childs Nerv Syst 24:815-820, 2008

23. Trenchs V, Curcoy AI, Navarro R, Pou J: Subdural haematomas and physical abuse in the first two years of life. Pediatr Neurosurg 43:352-357, 2007

24. Tucker J, Choudhary AK, Piatt J: Macrocephaly in infancy: benign enlargement of the subarachnoid spaces and subdural collections. J Neurosurg Pediatr 18:16-20, 2016

25. Tzioumi D, Oates RK: Subdural hematomas in children under 2 years. Accidental or inflicted? A 10-year experience. Child Abuse Negl 22:1105-1112, 1998

26. Vinchon M, Defoort-Dhellemmes S, Desurmont M, Dhellemmes P: Accidental and nonaccidental head injuries in infants: a prospective study. J Neurosurg 102 (4 Suppl):380-384, 2005

27. Williams RA: Injuries in infants and small children resulting from witnessed and corroborated free falls. J Trauma 31:1350-1352, 1991

28. Zahl SM, Egge A, Helseth E, Wester K: Benign external hydrocephalus: a review, with emphasis on management. Neurosurg Rev 34:417-432, 2011

\section{Disclosures}

The authors report no conflict of interest concerning the materials or methods used in this study or the findings specified in this paper.

\section{Author Contributions}

Conception and design: all authors. Acquisition of data: Fingarson, Ryan, Bregman. Analysis and interpretation of data: Ryan, McLone, Flaherty. Drafting the article: all authors. Critically revising the article: all authors. Reviewed submitted version of manuscript: Fingarson. Approved the final version of the manuscript on behalf of all authors: Fingarson. Statistical analysis: McLone.

\section{Correspondence}

Amanda Fingarson, Department of Pediatrics, Division of Child Abuse Pediatrics, Ann and Robert H. Lurie Children's Hospital of Chicago, 225 E Chicago Ave., Box 16, Chicago, IL 60611. email: afingarson@luriechildrens.org. 